\title{
Complex hydrogels based on aquasol and polyacrylamide
}

\author{
Chobit M., Kalin D., Tokarev V. \\ Institute of Chemistry and Chemical Technologies, Lviv Polytechnic National University, UKRAINE, Lviv, \\ 3/4 pl. Sviatoho Yura, e-mail: maksym.r.chobit@lpnu.ua
}

\begin{abstract}
The aim of the work is to obtain hydrogel composites, a crosslinked structure based on polyacrylamide and aquazole; to study the swelling kinetics of the obtained hydrogels and to study the thermomechanical properties of the obtained material.
\end{abstract}

Keywords - polymers, hydrogels, polymerization, aquazole, swelling.

In recent years, the development of chemical technologies has made it possible to obtain a large number of different polymers. One of the rapidly developing areas in this area is associated with polymeric hydrophilic gels (hydrogels). This type of polymer gels has a very high, compared to other materials, the ability to adsorb water, aqueous solutions, and other substances. They are biologically inactive, very similar to natural tissues due to the high water content. Due to these properties of polymer gels, they are actively used as fillers in diapers, surgical wipes, soft insoles. They are widely used in the chemical industry in the drainage of gases used in agriculture to obtain moisture-retaining soil. In medicine, hydrogels are used for the manufacture of contact lenses, for the treatment of wounds and skin burns, and are also considered promising for the use of polymer gels to create new generation implants. Hydrogels are also used in cosmetics to prevent wrinkles, namely hydrogel masks, which moisturize and fill skin cells with nutrients. Recently, the availability of hydrogels has increased significantly, and today, for example, hydrogel balls can be bought in almost any flower shop.

The chemistry of synthetic polymers opens up new possibilities for obtaining new types of hydrophilic polymers, architectures or combinations to improve their properties and future promising applications, in particular in the form of hydrogels. This class of materials has various interesting properties, individual shape and size, which is of great interest for biomedical, cosmetic use, biotechnology and other areas of human activity.

The aim of the work is to obtain hydrogel composites, a crosslinked structure based on polyacrylamide and aquazole (poly-2-ethyl-2-oxazoline) [1]; to study the swelling kinetics of the obtained hydrogels and to study the thermomechanical properties of the obtained material.

Based on the conducted studies, hydrogel composite materials based on crosslinked structures of polyacrylamide and aquazole were obtained. Aquazol has demonstrated excellent crosslinking properties of polymer materials. The degrees of swelling are calculated and thermomechanical properties are determined.

The degrees of swelling are calculated and thermomechanical properties are determined. The resulting hydrogel composites have high rates of swelling and are capable of long-term storage in an aqueous environment while maintaining elastic properties.

By grafted polymerization of hydrophilic functional monomers in an aqueous medium, spatially crosslinked polymer hydrogels containing chemically conjugated aquazole were obtained.

The process was performed in an aqueous solution of monomers (acrylamide (AAm) and acrylic acid (AA)) in the presence of the initiator $\mathrm{K}_{2} \mathrm{~S}_{2} \mathrm{O}_{8}$. A mixture of aquazole and an aqueous solution of monomers $(0.5 \mathrm{ml}$ in volume $)$ was intensively mixed using a magnetic stirrer.

After achieving homogenization, the reaction mixture was heated at $80^{\circ} \mathrm{C}$ for 5 hours. Polymerization was initiated by decomposition of the initiator $\mathrm{K}_{2} \mathrm{~S}_{2} \mathrm{O}_{8}$. 

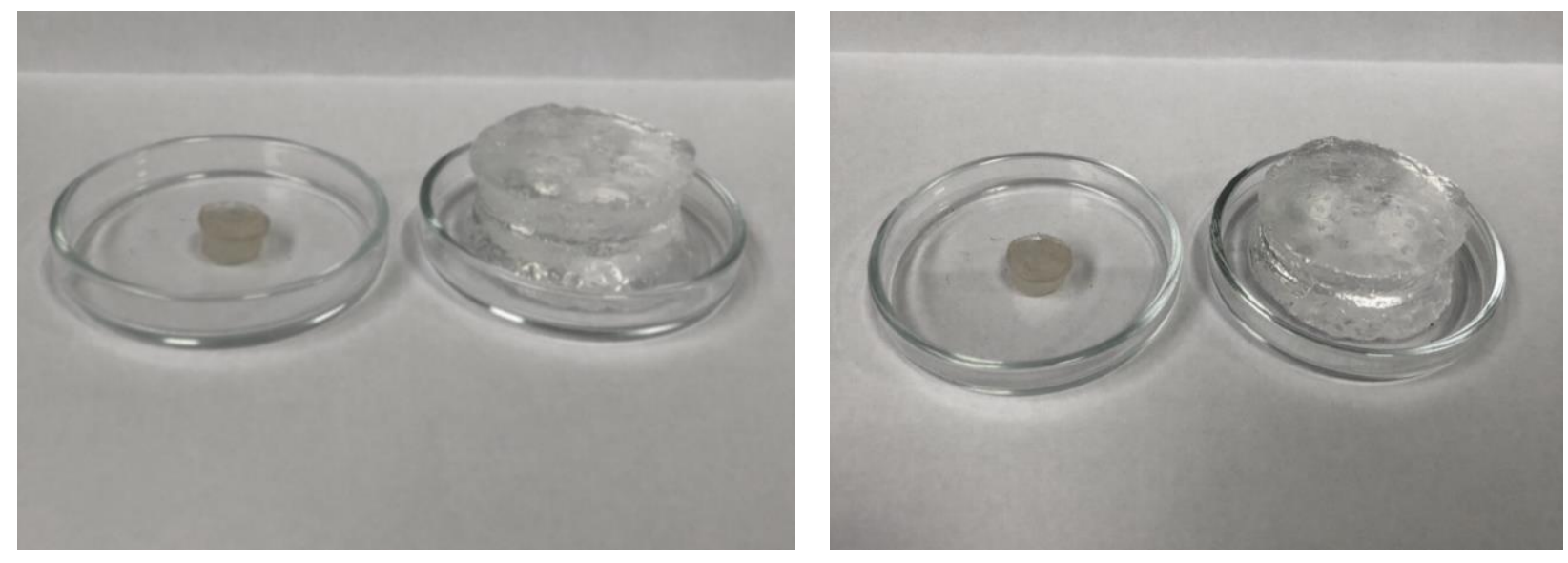

Fig.1. Hydrogels before and after prolonged exposure to water (copolymer composition, wt $\%$ : Aquazol = 20, AAm= 40, AA.=40).

\section{Conclusion}

New hydrogel composites based on polyacrylamide and copolymers of acrylamide and acrylic acid grafted with poly-2-ethyl-oxazoline were synthesized. Such composites show good absorbent properties, the degree of their swelling can reach more than $26,000 \%$ by weight. The study of the swelling kinetics of the obtained hydrogel samples showed that with an increase in the content of Aquazol 50, the degree of their swelling increases, and with an increase in the content of Aquazol 500, it decreases. Composites with Aquazol 500 show better absorbent properties compared to Aquazol 50 and are able to maintain their shape in an aqueous environment for a longer time. The dependences of swelling on the composition of the hydrogel composite indicate that when acrylic acid links are introduced into the hydrogel structure, the absorption capacity increases by half an order of magnitude. The kinetics of relative changes in the size of hydrogel composites during swelling was studied, which confirmed the main patterns obtained in the study of the kinetics of their swelling. The longest preservation of shape and size was observed for samples of Aquazol 500-based hydrogel composites containing acrylic acid links, apparently due to the fact that these composites have a stronger crosslinked structure compared to others. Thermomechanical studies of the obtained polymer materials were carried out and the temperature of their heat resistance was determined. It was found that with an increase in the content of Aquazoles, a natural decrease in the temperature of heat resistance is observed, and in the case of Aquazol 50 it is sharper. The introduction of AA links does not significantly affect the temperature of heat resistance of composites. From the conducted thermomechanical researches it is possible to draw a conclusion that composites have thermoplastic properties with rather low temperatures of heat resistance thanks to what it will be possible to process them easily into certain products.

\section{References}

[1] D. Pizzi, J. Humphries, J. P. Morrow, N. L. Fletcher, C. A. Bell, K. J. Thurecht, K. Kempe. (2019). Poly(2-oxazoline) macromonomers as building blocks for functional and biocompatible polymer architectures. European Polymer Journal. № 109258. P. 121. 